\title{
RSK1 promotes murine breast cancer growth and metastasis
}

\author{
Dominika Czaplinska ${ }^{1}$, Monika Gorska ${ }^{2}$, Kamil Mieczkowski ${ }^{2}$, Grazyna Peszynska-Sularz ${ }^{3}$, \\ Anna J. Zaczek ${ }^{1}$, Hanna M. Romanska ${ }^{4}$, Rafal Sadej ${ }^{2}$
}

${ }^{1}$ Department of Cell Biology, Intercollegiate Faculty of Biotechnology, University of Gdansk and Medical University of Gdansk, Gdansk, Poland

${ }^{2}$ Department of Molecular Enzymology, Intercollegiate Faculty of Biotechnology, University of Gdansk and Medical University of Gdansk, Poland

${ }^{3}$ Tri-City Academic Laboratory Animal Centre - Research and Services Centre, Medical University of Gdansk, Gdansk, Poland

${ }^{4}$ Department of Pathology, Medical University of Lodz, Lodz, Poland

\begin{abstract}
Introduction. Triple-negative breast cancer (TNBC), representing over 15\% of all breast cancers, has a poorer prognosis than other subtypes. There is no effective targeted treatment available for the TNBC sufferers. Ribosomal S6 kinases (RSKs) have been previously proposed as drug targets for TNBC based on observations that $85 \%$ of these tumors express activated RSKs.

Materials and methods. Herein we examined an involvement of RSK1 (p90 ribosomal S6 kinase 1) in a regulation of TNBC growth and metastatic spread in an animal model, which closely imitates human disease. Mice were inoculated into mammary fat pad with 4T1 cells or their RSK1-depleted variant. We examined tumor growth and formation of pulmonary metastasis. Boyden chamber, wound healing and soft agarose assays were performed to evaluate cells invasion, migration and anchorage-independent growth.

Results. We found that RSK1 promoted tumor growth and metastasis in vivo. After 35 days all animals inoculated with control cells developed tumors while in the group injected with RSK1-negative cells, there were 75\% tumor-bearing mice. Average tumor mass was estimated as $1.16 \mathrm{~g}$ and $0.37 \mathrm{~g}$ for RSK1-positive $v s$. -negative samples, respectively $(\mathrm{p}<0.0001)$. Quantification of the macroscopic pulmonary metastases indicated that mice with RSK1-negative tumors developed approximately $85 \%$ less metastatic foci on the lung surface $(\mathrm{p}<0.001)$. This has been supported by in vitro data presenting that RSK1 promoted anchorage-independent cell growth and migration. Moreover, RSK1 knock-down corresponded with decreased expression of cell cycle regulating proteins, i.e. cyclin D3, CDK6 and CDK4.

Conclusions. We provide evidence that RSK1 supports tumor growth and metastatic spread in vivo as well as in vitro migration and survival in non-adherent conditions. Further studies of RSK1 involvement in TNBC progression may substantiate our findings, laying the foundations for development of anti-RSK1-based therapeutic strategies in the management of patients with TNBC. (Folia Histochemica et Cytobiologica 2018, Vol. 56, No. 1, $11-20)$
\end{abstract}

Key words: RSK1 knock-down; triple-negative breast cancer; metastasis; animal model; in vitro; cell cycle regulation

\footnotetext{
Correspondecne address: Dr R. Sadej,

Department of Molecular Enzymology, Intercollegiate

Faculty of Biotechnology, University of Gdansk

and Medical University of Gdansk,

Gdansk 80-210, Poland

Dr HM Romanska, Department of Pathology,

Medical University of Lodz,

Lodz, 90-410, Poland

e-mail: rsadej@gumed.edu.pl,hanna.romanska@gmail.com
} 


\section{Introduction}

Triple-negative breast cancer (TNBC), defined by the lack of estrogen and progesterone receptors (ER, PR) as well as HER2 expression, represents approximately $15-20 \%$ of all breast cancers [1]. TNBC shows more aggressive clinical behavior with a high probability of local and distant metastases $[2,3]$. The majority of deaths take place within the first 5 years from initial diagnosis [4]. There is still no effective target-specific therapy for patients with TNBC and current regimens are based mainly on conventional chemotherapy (e.g. anthracyclines, taxanes, and platinum agents) [1,5]. Therefore, better knowledge of TNBC biology and identification of prognostic markers is an important clinical challenge.

Members of the $90 \mathrm{kDa}$ ribosomal S6 kinase (RSK) family of proteins regulate key cellular processes, such as migration, proliferation and survival [6]. Structurally, all four RSK isoforms (RSK1-4) share 73-80\% homology and their functional domains are highly-conserved. Despite these similarities, RSK1-4 may play various roles and their function appears to be cell-type specific $[6,7]$ since differences in their activation by upstream signaling and expression/subcellular localization patterns of individual RSKs as well as their specific preferences for substrates were reported [7]. We have previously demonstrated that FGF2/FGFR2 signaling activated RSK2 and that this pathway promoted in vitro anchorage-independent growth and migration of mammary epithelial and breast cancer cells [8]. Furthermore, we revealed RSK2-FGFR2 co-expression in breast cancer tissue and showed that patients with tumors expressing active RSK (RSK-P) alone or in combination with FGFR2 had worse disease-free survival [9]. Indeed, most studies show that RSK2 promotes cancer progression, whereas the role of RSK1 seems to be more controversial as it was shown to have both anti- and pro-metastatic effects depending on the cancer type $[6,7]$. Smith et al. reported that both RSK1 and RSK2 expression was elevated in approximately $50 \%$ of all breast tumors comparing to non-transformed tissues [10]. Genome-wide mRNA analysis of mammary epithelial cells revealed that RSK1 and RSK2 were major pro-migratory effectors of ERK/MEK pathway [11]. Accordingly, Smolen and colleagues demonstrated that RSK1 silencing prevented MCF10A cell migration in vitro [12]. Similar effect was observed in melanoma cells, where RSK1 overexpression influenced actomyosin stability resulting in increased cell motility [13]. In contrast, RSK1 decreased A549 lung cancer cells migration in vitro and their meta- static potential in a zebrafish model. Additionally, low RSK1 expression in primary tumors was shown to correlate with increased number of metastases in lung cancer patients [14]. Finally, RSK1 (contrary to RSK2) knock-down in head and neck squamous cell carcinoma (HNSCC) cells did not influence invasiveness at all [15].

Despite the continuous research in the field, functions of the RSK isoforms are still not well understood. Several authors have suggested that RSK1 plays an important role in TNBC, in particular in regulating cell motility and invasiveness [16-18]; however, the significance of RSK1 for the regulation of TNBC metastasis has never been investigated. Therefore, we examined for the first time an involvement of RSK1 in the regulation of TNBC growth and progression in an animal model which exhibits similar metastatic pattern to that of human disease.

\section{Materials and methods}

Cell culture and reagents. Murine triple-negative breast cancer $4 \mathrm{~T} 1$ cell line was obtained from ATCC. Cells were grown at $37^{\circ} \mathrm{C}$ in a humidified atmosphere of $5 \% \mathrm{CO}_{2}$ in DMEM supplemented with $10 \%$ fetal bovine serum (FBS) and penicillin/streptomycin $(100 \mathrm{U} / \mathrm{mL} / 100 \mu \mathrm{g} / \mathrm{mL})$. Media and their supplements were purchased from Sigma-Aldrich (St. Louis, Missouri, USA). RIPA Buffer 10X (\#9806) and the following antibodies were obtained from Cell Signaling Technology (Danvers, MA, USA): anti-Akt (\#9272), anti-Akt-Ser473 (\#4058), anti-Bax (\#5023), anti-Bcl-xl (\#2762), anti-cyclin D1 (\#2978), anti-cyclin D3 (\#2936), anti-CDK2 (\#2546), anti-CDK4 (\#12790), anti-CDK6 (\#3136), anti-cytokeratin 7 (\#4465), anti-cytokeratin 17 (\#4543), anti-cytokeratin 8/18 (\#4546), anti-cytokeratin 18 (\#4548), anti-cytokeratin 19 (\#4558), anti-Pan-keratin (\#4545), anti-E-cadherin (\#14472), anti-ERK1/2 (\#4695), anti-ERK1/2-Thr202/Tyr204 (\#9101), anti-FAK (\#3285), anti-FAK-Tyr397 (\#3283), anti-p38 (\#9212), anti-p38-Thr180/Tyr182 (\#9211), anti-RSK1 (\#8408), anti-RSK2 (\#5528), anti-SAPK/JNK-Thr183/Tyr185 (\#9251) anti-Src (\#2108) and anti-Src-Tyr416 (\#2101). Antibodies against Bcl-2 (M0887) were from Dako (Glostrup, Denmark). Antibodies anti- $\beta$-actin (A5316), anti-vimentin (V6389) and low gelling temperature agarose (A9414) were obtained from Sigma-Aldrich. Goat anti-rabbit (926-68021) and Donkey anti-mouse (926-32212) IRDye secondary antibodies were purchased from LI-COR Biosciences (Lincoln, NE, USA).

Establishment of RSK1 knock-down. 4T1 RSK1-negative cell line was generated with lentiviral system based on pLKO.1-TRC vector (Addgene, Cambridge, Massachusetts, USA, \#10878) with cloned shRNA designed on the basis of the following DNA sequence: 5'- CCC AAC ATC ATC 
ACT CTG AAA -3' [19]. 4T1 CTRL cell line was transfected with pLKO.1 backbone plasmid and used as a control. Transfection was done using TurboFect Transfection Reagent (Thermo Scientific, Waltham, MA, USA) according to the manufacturer's protocol. Stable cell line expressing shRNA was established by puromycin selection. RSK1 knock-down efficiency was verified by Western blotting.

Animal experiments. BALB/c mice (5-week-old females) were purchased from and maintained in the Tri-City Academic Laboratory Animal Centre - Research and Services Centre of the Medical University of Gdansk, Poland. Animals were maintained on a regular light-dark cycle at $22 \pm 2^{\circ} \mathrm{C}$ and provided with freely available food and water. $10^{5}$ cells in $50 \mu \mathrm{l}$ of phosphate-buffered saline (PBS) were injected into mammary fat pad. 12 mice received RSK1-negative cells while the control group $(\mathrm{N}=12)$ was injected with control, RSK1-positive cells. Growing tumors were measured every three days by digital caliper starting on day 8 after cancer cells transplantation. After sacrificing the animals by cervical dislocation on day 35 primary tumors were removed, measured and weighted. Their volume was calculated with the formula of an ellipsoid (length $\times$ width $\times$ height $\times 0.5236$ ) [20]. Lungs were collected and visually examined for the number of macrometastases by two investigators independently [21,22]. This study was conducted in accordance with the Local Ethics Committee of the Medical University of Gdansk, Poland (permission number: 46/2015, obtained 26/10/2015).

Western blotting. Cells grown up to $80-90 \%$ of confluency were lysed with $2 \times$ concentrated Laemmli buffer containing $2 \mathrm{mM}$ PMSF, $10 \mu \mathrm{g} / \mathrm{ml}$ aprotinin, $10 \mu \mathrm{g} / \mathrm{ml}$ leupeptin, $5 \mathrm{mM}$ EGTA, $1 \mathrm{mM}$ EDTA, $2 \mathrm{mM} \mathrm{Na}_{4} \mathrm{P}_{2} \mathrm{O}_{7}, 5 \mathrm{mM} \mathrm{NaF}$ and $5 \mathrm{mM}$ $\mathrm{Na}_{3} \mathrm{VO}_{4}$. Tissue specimens were homogenized with MagNA Lyser (Roche, Basel, Switzerland) and lysed in $1 \times$ RIPA buffer supplemented with all inhibitors. Samples with equal amounts of protein per lane (at the range of 20-30 $\mu \mathrm{g}$ ) were resolved by SDS polyacrylamide gel electrophoresis (SDS-PAGE) and transferred onto nitrocellulose membrane. The membranes were incubated for $1 \mathrm{~h}$ in $5 \%$ skimmed milk in TBST and probed with specific antibodies overnight at $4{ }^{\circ} \mathrm{C}$ followed by $1 \mathrm{~h}$ incubation with IRDye secondary antibodies. Specific protein bands were visualized with Odyssey ${ }^{\circledR}$ CLx Imaging System (LI-COR Biosciences) according to the manufacturer's protocol.

Soft agarose assay for anchorage-independent growth. Soft agarose assay was performed as described before [8, 23]. Briefly, $10^{4}$ cells per well of 6 -well plate were suspended in $3 \mathrm{ml}$ of $0.5 \%$ low gelling temperature agarose prepared in DMEM (10\% FBS) and overlaid on $3 \mathrm{ml}$ solidified $0.6 \%$ bottom layer of agarose. The top layer was covered with $3 \mathrm{ml}$ of DMEM (10\% FBS) refreshed every 2-3 days. After
10 days of culture colonies were counted and measured using PrimoVert microscope (Carl Zeiss, Jena, Germany) and ImageJ software developed at the National Institutes of Health (Bethesda, MD, USA).

Migration assay. 4T1 cells were detached using Cell Dissociation Solution (Millipore, Billerica, MA, USA). The $5 \times$ $10^{5}$ cell were suspended in serum-free DMEM and placed in the inner compartment of Boyden chamber inserts with polycarbonate membranes $(8-\mu \mathrm{m}$ pores, BD Biosciences, Franklin Lakes, NJ, USA), pre-coated with $50 \mu$ l Matrigel Matrix Growth Factor Reduced (BD Biosciences) diluted 1:100 with serum-free media. Cells were allowed to migrate toward DMEM containing 10\% FBS. After $24 \mathrm{~h}$ non-migrated cells were removed by cotton swab. Nuclei of migrated cells were stained with DAPI and counted using a AxioVert 200 microscope (Carl Zeiss). Ten to twenty random fields per membrane were photographed and analyzed in each experiment.

Wound healing assay. 4T1 cells were plated in a 6-cm culture dish and incubated at $37^{\circ} \mathrm{C}$. The following day scratches were made by scraping confluent monolayers with a sterile pipette tip in the center of the dish. Next, the cells were washed twice with PBS and incubated overnight in serum-free media. Subsequently, media containing $10 \%$ FBS was added to initiate cell motility. To measure the migration capacities, images were captured at the beginning of the experiment and $6 \mathrm{~h}$ after wounding. The wound closure was evaluated using Image J software.

Proliferation assay. Proliferation tests were performed using WST-1 reagent (Roche) in accordance with manufacturer's protocol. Briefly, 4T1 control and RSK1-negative cells were cultured in 96-well microplates. After 96 h WST-1 was added to the cells and incubated for next $4 \mathrm{~h}$. Cell viability was determined by the absorbance measurement at $440 \mathrm{~nm}$.

Statistical analyses. All data are expressed as means \pm SD from at least three independent experiments. Comparative data were analyzed with Mann-Whitney U test using the Statistica 10.0 software (Statsoft, Cracow, Poland). The results were considered statistically significant when $p$ value was lower than 0.05 .

\section{RESULTS}

\section{RSK1 promotes primary tumor growth and lung metastasis in vivo}

To investigate the functional significance of RSK1 expression in breast cancer growth and metastasis, we generated stable knock-down of RSK1 in 4T1 cell line using a pLKO.1-TRC vector expressing spe- 


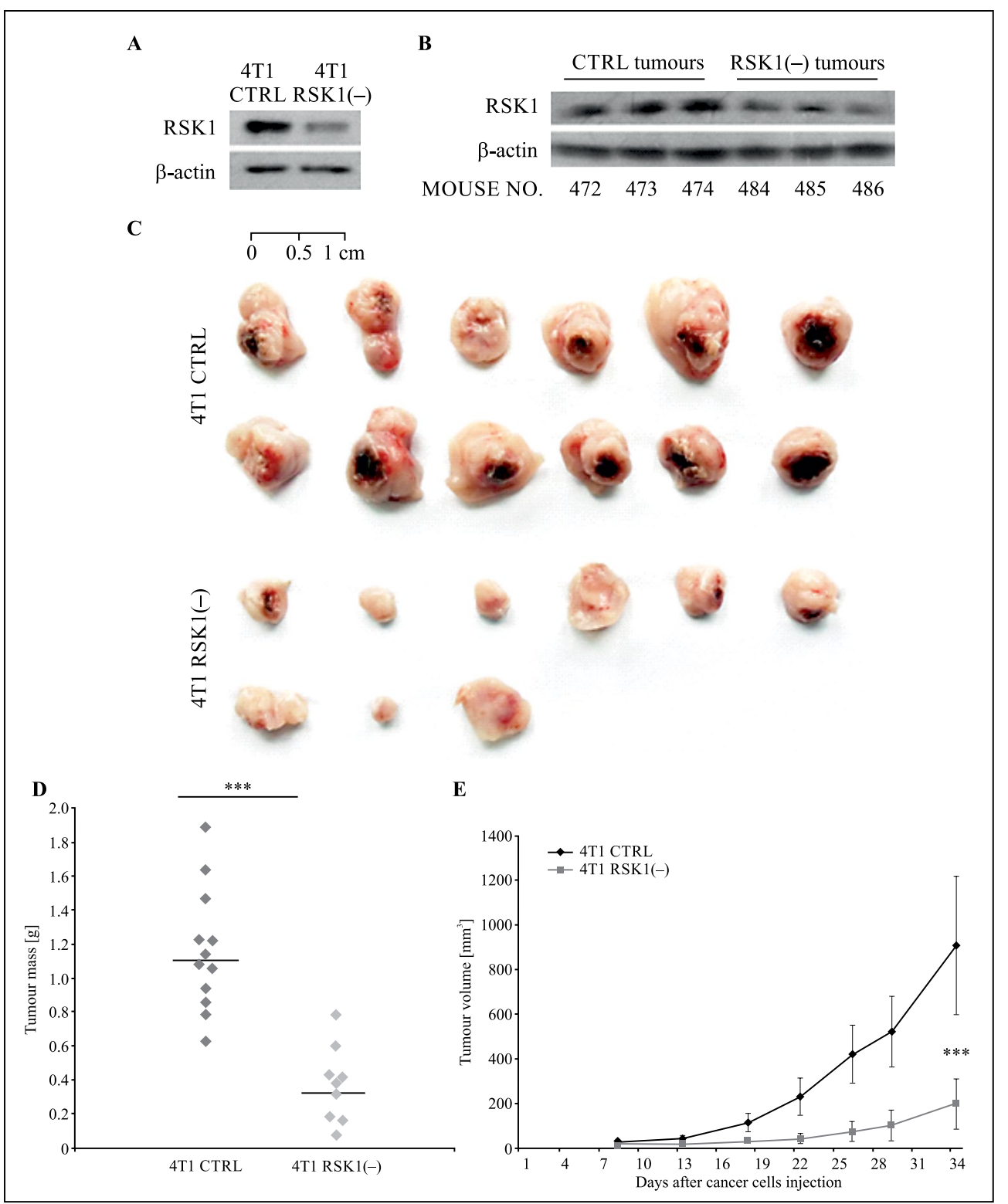

Figure 1. RSK1 promotes tumor growth in vivo. A. Stable knock-down of RSK1 in 4T1 cells. B. RSK1 expression in tumors resected at the $35^{\text {th }}$ day of growth. Cells were injected into mammary fat pads of BALB/c mice. C. All animals injected with wild-type 4T1 cells $(\mathrm{N}=12)$ developed tumors while in the second group $(\mathrm{N}=12)$ injected with RSK1-negative cells there were only 9 tumor-bearing mice. D. Average tumor mass was $1.16 \mathrm{~g} \pm 0.36$ and $0.37 \mathrm{~g} \pm 0.22$ for control and RSK1-negative tumors respectively, ${ }^{* * *} \mathrm{p}<0.0001$. E. Tumor size was measured every three days starting on day 8 after cancer cell implantation. The volumes of tumors were calculated using the formula of an ellipsoid (length $\times$ width $\times$ height $\times 0.5236$ ). $* * * \mathrm{p}<0.0001$ refers to statistical analysis for the last measurement, on $34^{\text {th }}$ day). Each square represents individual tumor. Straight lines represent the average value for each group.

cific shRNA sequence targeting RSK1 (designed on previously published siRNA [19], shown to lack any off-target effects). A significant reduction in RSK1 protein level as compared to the control (transfected with pLKO.1 backbone plasmid) was shown by Western blotting (Fig. 1A). RSK1 knock-down did not induce any phenotypic changes in 2-dimensional culture and RSK1-negative variant exhibited the same proliferation rate in vitro as 4T1 control cells (Fig. 2). Both 4T1 variants i.e. control and RSK1-negative were orthotopically implanted into mammary fat pads of BALB/c mice. Tumors were palpable 6-10 days after cell inoculation. Stability of RSK1 knock-down was confirmed in tumors resected after 35 days from the primary sites (Fig. 1B). The depletion of RSK1 led to a significant reduction in primary tumor growth. All 


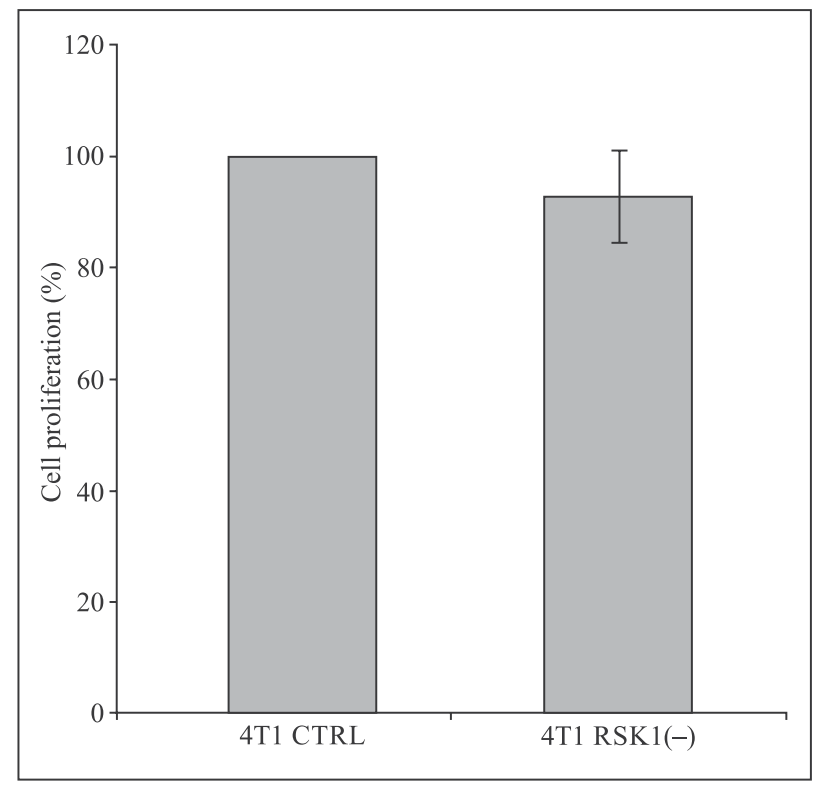

Figure 2. Proliferation rates of 4T1 CTRL and RSK1-negative cells. The values presented are means $\pm \mathrm{SD}(\mathrm{N}=3), \mathrm{p}=$ $=0.98$. After $96 \mathrm{~h}$ of growth WST-1 reagent was added to the cells and incubated for next $4 \mathrm{~h}$. Cell viability was determined by the absorbance measurement at $440 \mathrm{~nm}$.

mice in control group $(\mathrm{N}=12)$ developed tumors, while in the second group $(\mathrm{N}=12)$ that contained animals injected with RSK1-negative cells there were only 9 tumor-bearing animals (Fig. 1C). Overall tumor size and mass were lower in the RSK1-depleted cohort compared to the control with average value of $0.37 \mathrm{~g}$ and $1.16 \mathrm{~g}$, respectively ( $<$ 0.0001) (Fig. 1D-E). As expected from the above data, RSK1 knock-down affected formation of distant metastases. $58 \%$ of the animals transplanted with RSK1-negative cells displayed colonization in lungs compared to $100 \%$ of the mice in the control group. Quantification of the macroscopic pulmonary metastases (as described previously in $[21,22,24]$ ) indicated that RSK1-depleted cohort developed an average 2.83 metastatic foci on the lung surface compared with 18.25 foci in the control cohort ( $\mathrm{p}<0.001)$ (Fig. 3A-B).

\section{RSK1 increases migration in vitro}

To investigate in vitro the functional consequences of RSK1 depletion in 4T1 cells, we assessed their potential to grow in anchorage-independent conditions. 4T1 CTRL and RSK1-negative cells were cultured in soft-agarose for 10 days. RSK1 silencing reduced size of the colonies (which reflects proliferation rate in anchorage-independent conditions) by nearly $40 \%$ $(\mathrm{p}<0.001)$ and its clonogenic potential by approximately $60 \%$ (p < 0.0001) (Fig. 4A-C). To determine if RSK1

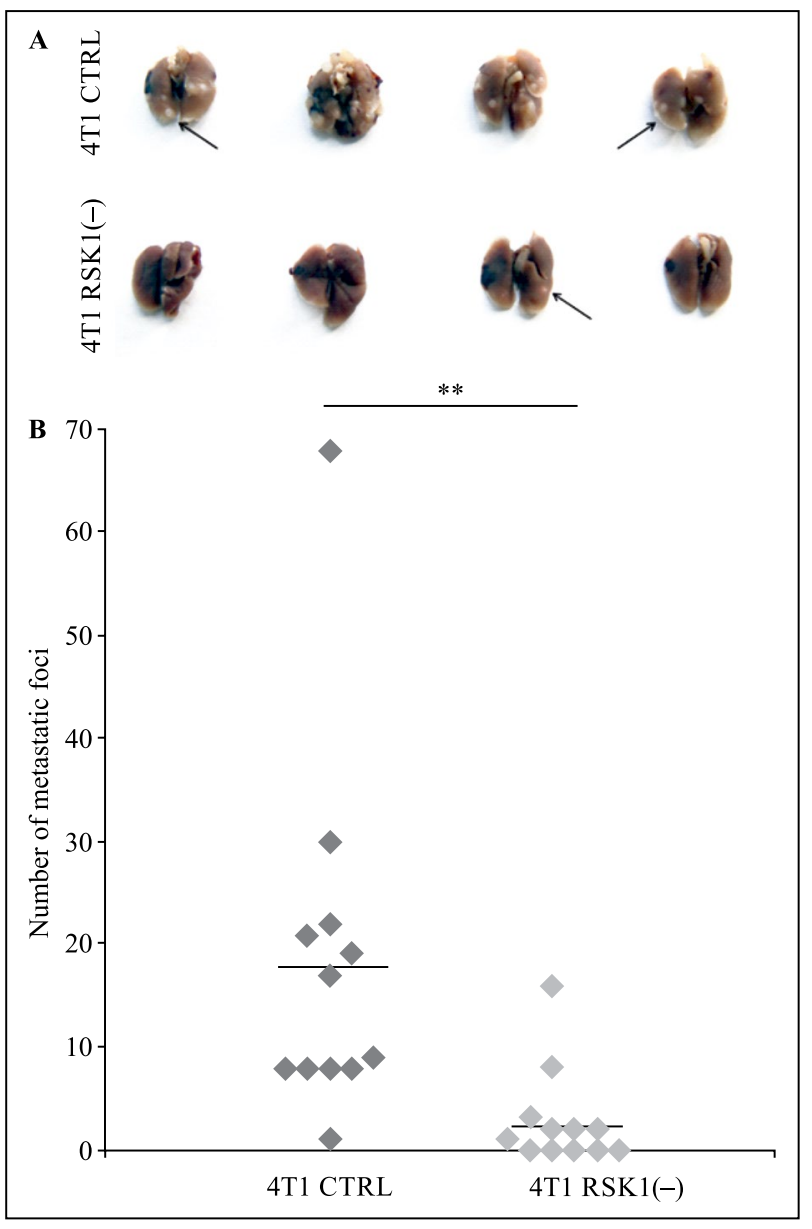

Figure 3. RSK1 promotes pulmonary metastasis. A. Lungs were resected 35 days after cancer cells injection into mammary fat pads. Representative examples of the macroscopic metastatic foci (arrows) on the lung surface of mice bearing RSK1-positive (upper panel) and RSK1-negative mammary tumors (lower panel). B. Quantification of the macroscopic pulmonary metastases indicated that mice with RSK1-negative tumors developed on average $2.83( \pm 4.73)$ metastatic foci on the lung surface compared with 18.25 ( \pm 17.68) foci in the group with control tumors. Each square represents individual mice. Straight lines represent the average value for each group, ${ }^{* *} \mathrm{p}<0.001$.

knock-down affects 4T1 cell motility and invasion, the Boyden chamber migration assay and wound healing test were performed. As shown in Figure 5A-B, silencing of RSK1 reduced trans-well migration of 4T1 RSK1 cells by nearly $50 \%$ ( $\mathrm{p}<0.0001)$. Migration rates were also evaluated by measuring the distance between edges of the "wound". Six hours after scratching, 4T1 RSK1-negative cells showed significantly reduced migration abilities. On the contrary, 4T1 CTRL cells almost completely healed up, covering approximately $70 \%$ of the "wound" width comparing to the $40 \%$ in RSK1-depleted cells $(\mathrm{p}<0.05)$ (Fig. 5C-D). 


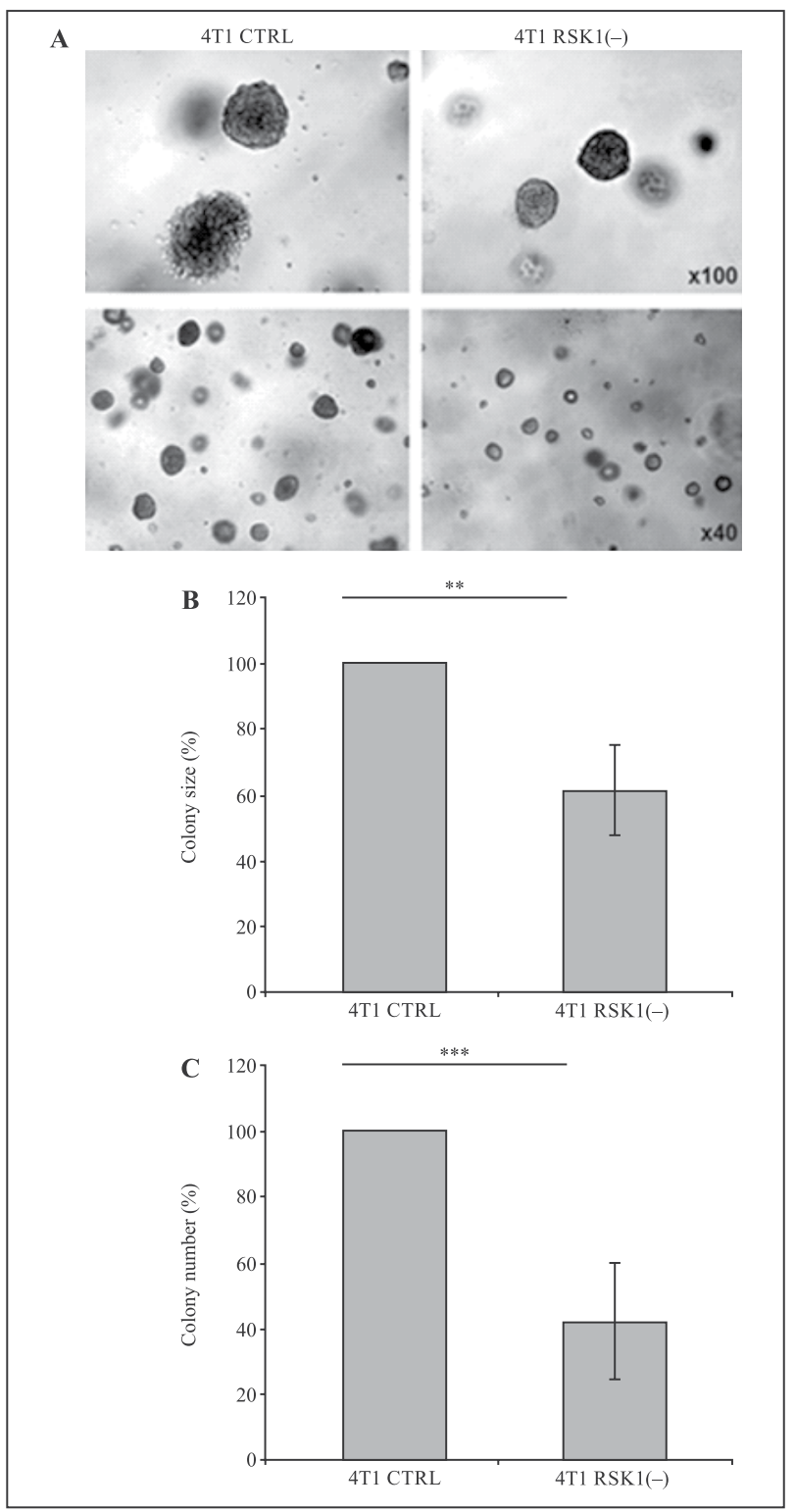

Figure 4. RSK1 promotes anchorage-independent growth of 4T1 cells in vitro. A. 4T1 cells growth in soft agarose. Microphotographs were taken after 10 days of culture. B. Measurement of colonies volume was done with ImageJ software. RSK1 knock-down in 4T1 cells reduced colony volume by nearly $40 \%,{ }^{* *} \mathrm{p}<0.001$. C. The average percentage of colonies formed in soft agarose, ${ }^{* * *} \mathrm{p}<0.0001$. The values presented are means $\pm \mathrm{SD}(\mathrm{N}=3)$.

\section{RSK1 regulates Src activity and expression of cell cycle regulating proteins}

To define molecular mechanism of RSK1 role in breast cancer progression, we analyzed a panel of signaling molecules that play a critical role in controlling growth, apoptosis and cell migration. Western blotting analysis revealed no convincing RSK1-dependent differences in the level of the activation of pro-pro- liferative kinases such as Akt, p38, ERK1/2 or SAPK/ /JNK (Fig. 6A). However, RSK1 knock-down corresponded with decreased Src activity. Furthermore, in 4T1 RSK1-negative cells, we observed a significant decrease in the level of cell cycle regulating proteins: cyclin D3 and CDK6 accompanied with a reduction of CDK4 expression ( $p<0.05)$, without changes in cyclin D1 and CDK2 expression (Fig. 6A-B). In addition, RSK1 silencing had no effect on the expression of proteins involved in apoptosis (Bcl-2, Bax, Bcl-Xl) (Fig. 6A). There was also no change in the expression of cytokeratins (CK7, CK8, CK17, CK18, CK19) and epithelial-mesenchymal transition (EMT) markers (E-cadherin, vimentin) (Fig. 6A). Therefore, we conclude that Src kinase seems to be involved in RSK1-dependent cell motility, whereas cyclin D3 may be engaged in RSK1-mediated cell growth.

\section{Discussion}

Murine 4T1 triple-negative breast cancer cells closely imitate human disease when inoculated into mammary fat pad. Moreover, unlike most of the in vivo breast cancer models, 4T1 cells can spontaneously metastasize from the primary site to lungs, bones, brain and liver with a frequency analogous to human disease [25]. Therefore we chose 4T1 cell line to investigate for the first time the involvement of RSK1 in the TNBC development and metastasis and found that RSK1 promoted primary tumor growth. Depletion of RSK1 resulted in over three times smaller tumors and strongly decreased number of metastatic foci on the lung surface.

Several studies have already implied that RSK1 plays an important role in breast cancer, in particular emphasizing its function in regulating cell motility [11, 12]. Stratford et al. concluded that RSK1 and RSK2 are key activators of a transcription factor YB-1 in human TNBC. They found the EGFR > RSK1/2 > $>$ YB-1 pathway as the main driver of TNBC which is thought to contribute to cells aggressive behavior [16]. These results are in line with a study showing that RSK1 and RSK2 promoted the TNBC cells (MDA-MB-231 cell line) metastatic spread to liver in vivo. Silencing of RSK1 reduced the number of metastatic foci to the similar extend as shown in our study. Furthermore, the level of activated RSKs was higher in TNBC tumors than in non-transformed mammary tissue [18]. Additionally, it was demonstrated that growth and migration of MDA-MB-231 TNBC cells, contrary to ER/PR-positive MCF7 cells, depended on both RSK1 and RSK2 activity [26]. RSKs have been previously proposed as drug targets for TNBC based on observations that $85 \%$ of these tumors expressed 


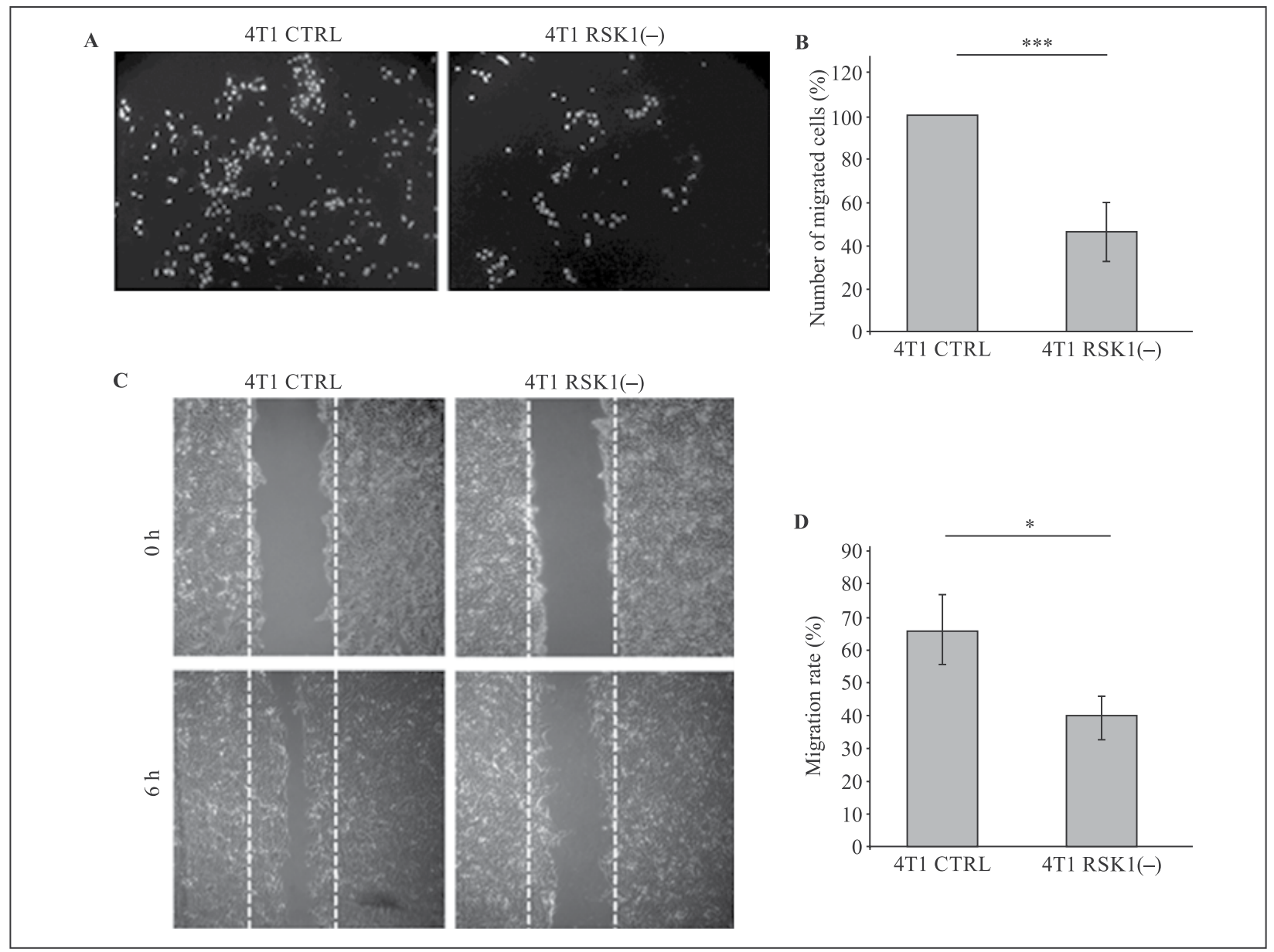

Figure 5. RSK1 enhances 4T1 cells migration in vitro. A. Representative images of Boyden chamber membranes. Nuclei of migrated cells were stained with DAPI. B. 4T1 control and RSK1-negative cells migration rate in Boyden chamber assay, $* * * \mathrm{p}<0.0001$. C. Representative images from the wound-healing assay taken 0 and $6 \mathrm{~h}$ after scratching. D. Bar graphs showing wound closure rates in 4T1 control and RSK1-negative cells ${ }^{*} \mathrm{p}<0.05$. The values presented are means \pm SD $(\mathrm{N}=3)$.

activated RSKs [17]. There are also reports suggesting that RSK1 behaves as a tumor suppressor. Zhao et al. demonstrated that level of RSK1 was lower in breast cancer tumors compared to adjacent tissue. RSK1 mRNA expression was inversely correlated with tumor size, stage and metastasis to nodes. In addition patients with decreased RSK1 transcript had shorter DFS and OS. Surprisingly, the same study showed that selective RSK inhibitor SL0101 decreased invasion of MDA-MB-231 TNBC cells [27]. Taking into account this and our data it would be interesting to stratify patient cohorts into individual subtypes and examine prognostic significance of RSK1 for TNBC women.

Resistance to anchorage dependence and ability to migrate are hallmarks of metastatic cancers. We showed that RSK1 silencing impaired anchorage-independent growth and motility of 4T1 cells. Our findings are partially in line with those of Cuesta and Holz, who reported that both RSK1 and RSK2 are required for MDA-MB-231 cells growth in anchorage-dependent clonogenic assay. Contrary to our observations, individual silencing of RSK1 or RSK2 did not suppressed cell proliferation, suggesting a compensatory role of both isoforms in MDA-MB-231 cell line what seems to be not relevant for 4T1 cells [26]. We also found that RSK1 modulated activity of Src kinase shown to promote survival and invasion of cancer cells [28]. We believe that decreased Src phosphorylation in RSK1-negative 4T1 cells may be responsible for their limited metastatic potential. In addition, we observed that knock-down of RSK1 triggered significant reduction of the levels of proteins that are involved in cell cycle regulation, i.e. cyclin D3 and its partner CDK6, accompanied with a reduction of CDK4 expression $(p<0.05)$. This is contrary to the findings showing that RSK1 and RSK2 through the regulation of translation repressor PDCD4 reduced the levels of cyclin D1 and Bcl-2 what consequently 


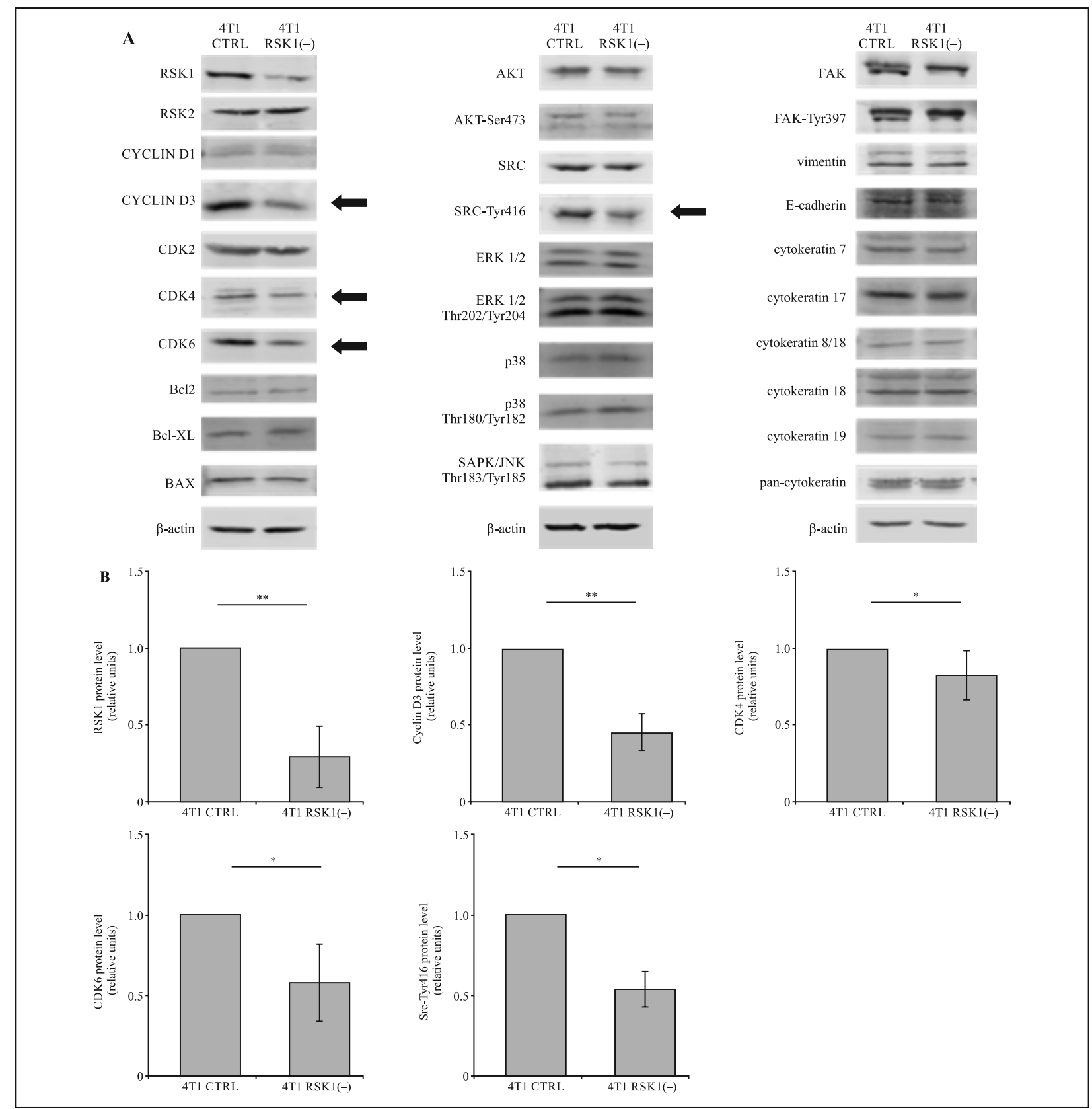

Figure 6. Western blotting analysis of control and RSK1-negative 4T1 cells. A. We compared expression and activation of indicated proteins involved in proliferation (Akt, p38, ERK1/2, SAPK/JNK, Src), cell cycle regulation (cyclin D1, cyclin D3, CDK2, CDK4, CDK6), apoptosis (Bcl-2, Bax, Bcl-Xl), epithelial-to-mesenchymal transition (E-cadherin, vimentin) and cytoskeleton formation (CK7, CK8, CK17, CK18, CK19). Experiments were done in triplicates. B. Densitometry analysis of selected Western blots presented above. ${ }^{*} \mathrm{p}<0.05,{ }^{* *} \mathrm{p}<0.001$. Analysis was done with ImageJ software.

induced apoptosis in MDA-MB-231 cells [26]. Discrepancies between these results may be explained by the fact that Cuesta and Holz used double knockdown of both isoforms and RSKs pan-inhibitor while our study focused specifically on RSK1 silencing. This is supported by studies which reported that RSK2, but not RSK1, is the key regulator of cyclin D1 expression in various cancer cell lines $[29,30]$.
Accordingly to our results, Chi et al. indicated that cyclin D3 expression was associated with poor DFS of breast cancer patients. Protein and mRNA levels of cyclin D3 were up-regulated in both mammary tumors and breast cancer cell lines. Furthermore, cyclin D3 was shown to physically interact with actin in MCF-7 and MDA-MB-231 cells and enhance breast cancer cell migration and invasion in vitro [31]. This may 
explain our observation of decreased D3 expression in RSK1-negative 4T1 cells. 4T1 RSK1-negative cells displayed decreased expression of cell cycle proteins, although their 2D proliferation had not been affected. It is known that cyclin expression level not always correlates with the proliferation rate. In breast cancer patients cyclin D3, a predictor of better DFS, displayed no correlation with widely used proliferation marker Ki67 [31]. Moreover, Sicinska et al. showed that knock-down of cyclin D3 had no impact on proliferation of leukemia cell lines [32]. Our present results suggest that cyclin D3 is engaged only in RSK1-mediated 3-dimensional growth in soft agarose growth (but not in 2D growth) of 4T1 cells, probably because of an additional pressure cells are subjected in anchorage-independent conditions.

To the best of our knowledge, this is the first report on RSK1 role in a clinically relevant animal model of TNBC. We provide evidence that RSK1 supports tumor growth and metastatic spread in vivo as well as in vitro migration and survival in non-adherent conditions.

\section{Acknowledgements}

This paper was supported by the following grants from National Science Center: UMO-2014/15/N/ /NZ3/04305 (to DC) and UMO-2016/23/N/NZ3/02927 (to KM).

First author's (DC) PhD scholarships were provided by Polpharma Scientific Foundation (2017) and National Science Center (Etiuda no UMO-2017/24/T/ (NZ3/00082).

\section{Conflict of interest}

The authors declared no conflicts of interest.

\section{References}

1. Jerusalem G, Collignon J, Schroeder H, et al. Triple-negative breast cancer: treatment challenges and solutions. Breast Cancer: Targets and Therapy. 2016: 93, doi: 10.2147/bctt.s69488.

2. Irvin WJ, Carey LA. What is triple-negative breast cancer? Eur J Cancer. 2008; 44(18): 2799-2805, doi: 10.1016/j. ejca.2008.09.034, indexed in Pubmed: 19008097.

3. Lin NU, Claus E, Sohl J, et al. Sites of distant recurrence and clinical outcomes in patients with metastatic triple-negative breast cancer: high incidence of central nervous system metastases. Cancer. 2008; 113(10): 2638-2645, doi: 10.1002/ /cncr.23930, indexed in Pubmed: 18833576.

4. Dent R, Trudeau M, Pritchard KI, et al. Triple-negative breast cancer: clinical features and patterns of recurrence. Clin Cancer Res. 2007; 13(15): 4429-4434, doi: 10.1158/1078-0432. CCR-06-3045, indexed in Pubmed: 17671126.

5. Hudis CA, Gianni L. Triple-negative breast cancer: an unmet medical need. Oncologist. 2011; 16 Suppl 1: 1-11, doi: 10.1634/ /theoncologist.2011-S1-01, indexed in Pubmed: 21278435.
6. Sulzmaier FJ, Ramos JW. RSK isoforms in cancer cell invasion and metastasis. Cancer Res. 2013; 73(20): 6099-6105, doi: 10.1158/0008-5472.CAN-13-1087, indexed in Pubmed: 24097826.

7. Lara R, Seckl MJ, Pardo OE. The p90 RSK family members: common functions and isoform specificity. Cancer Res. 2013; 73(17): 5301-5308, doi: 10.1158/0008-5472.CAN-12-4448, indexed in Pubmed: 23970478.

8. Czaplinska D, Turczyk L, Grudowska A, et al. Phosphorylation of RSK2 at Tyr529 by FGFR2-p38 enhances human mammary epithelial cells migration. Biochim Biophys Acta. 2014; 1843(11): 2461-2470, doi: 10.1016/j.bbamcr.2014.06.022, indexed in Pubmed: 25014166.

9. Czaplinska D, Mieczkowski K, Supernat A, et al. Interactions between FGFR2 and RSK2-implications for breast cancer prognosis. Tumour Biol. 2016; 37(10): 13721-13731, doi: 10.1007/s13277-016-5266-9, indexed in Pubmed: 27476168.

10. Smith JA, Poteet-Smith CE, Xu Y, et al. Identification of the first specific inhibitor of p90 ribosomal S6 kinase (RSK) reveals an unexpected role for RSK in cancer cell proliferation. Cancer Res. 2005; 65(3):1027-1034, indexed in Pubmed: 15705904.

11. Doehn U, Hauge C, Frank SR, et al. RSK is a principal effector of the RAS-ERK pathway for eliciting a coordinate promotile/invasive gene program and phenotype in epithelial cells. Mol Cell. 2009; 35(4): 511-522, doi: 10.1016/j.molcel.2009.08.002, indexed in Pubmed: 19716794.

12. Smolen GA, Zhang J, Zubrowski MJ, et al. A genome-wide RNAi screen identifies multiple RSK-dependent regulators of cell migration. Genes Dev. 2010; 24(23): 2654-2665, doi: 10.1101/gad.1989110, indexed in Pubmed: 21062900.

13. Larrea MD, Hong F, Wander SA, et al. RSK1 drives p27Kip1 phosphorylation at T198 to promote RhoA inhibition and increase cell motility. Proc Natl Acad Sci U S A. 2009; 106(23): 9268-9273, doi: 10.1073/pnas.0805057106, indexed in $\mathrm{Pu}-$ bmed: 19470470.

14. Lara R, Mauri FA, Taylor H, et al. An siRNA screen identifies RSK1 as a key modulator of lung cancer metastasis. Oncogene. 2011; 30(32): 3513-3521, doi: 10.1038/onc.2011.61, indexed in Pubmed: 21423205.

15. Kang S, Elf S, Lythgoe K, et al. p90 ribosomal S6 kinase 2 promotes invasion and metastasis of human head and neck squamous cell carcinoma cells. J Clin Invest. 2010; 120(4): 1165-1177, doi: 10.1172/JCI40582, indexed in Pubmed: 20234090.

16. Stratford AL, Fry CJ, Desilets C, et al. Y-box binding protein-1 serine 102 is a downstream target of p90 ribosomal S6 kinase in basal-like breast cancer cells. Breast Cancer Res. 2008; 10(6): R99, doi: 10.1186/bcr2202, indexed in Pubmed: 19036157.

17. Stratford AL, Reipas K, Hu K, et al. Targeting p90 ribosomal S6 kinase eliminates tumor-initiating cells by inactivating Y-box binding protein-1 in triple-negative breast cancers. Stem Cells. 2012; 30(7): 1338-1348, doi: 10.1002/stem.1128, indexed in Pubmed: 22674792.

18. Ludwik KA, Campbell JP, Li M, et al. Development of a RSK Inhibitor as a Novel Therapy for Triple-Negative Breast Cancer. Mol Cancer Ther. 2016; 15(11): 2598-2608, doi: 10.1158/1535-7163.MCT-16-0106, indexed in Pubmed: 27528706.

19. Shahbazian D, Roux PP, Mieulet V, et al. The mTOR/ PI3K and MAPK pathways converge on eIF4B to control its phosphorylation and activity. EMBO J. 2006; 25(12): 2781-2791, doi: 10.1038/sj.emboj.7601166, indexed in Pubmed: 16763566 
20. Kaur P, Nagaraja GM, Zheng H, et al. A mouse model for triple-negative breast cancer tumor-initiating cells (TNBC-TICs) exhibits similar aggressive phenotype to the human disease. BMC Cancer. 2012; 12: 120, doi: 10.1186/1471-2407-12-120, indexed in Pubmed: 22452810.

21. Ogino H, Hanibuchi M, Kakiuchi S, et al. E7080 suppresses hematogenous multiple organ metastases of lung cancer cells with nonmutated epidermal growth factor receptor. Mol Cancer Ther. 2011; 10(7): 1218-1228, doi: 10.1158/1535-7163. MCT-10-0707, indexed in Pubmed: 21551260.

22. Cai WB, Zhang Y, Cheng R, et al. Dual inhibition of plasminogen kringle 5 on angiogenesis and chemotaxis suppresses tumor metastasis by targeting HIF- $1 \alpha$ pathway. PLoS One. 2012; 7(12): e53152, doi: 10.1371/journal.pone.0053152, indexed in Pubmed: 23300882.

23. Piasecka D, Kitowska K, Czaplinska D, et al. Fibroblast growth factor signalling induces loss of progesterone receptor in breast cancer cells. Oncotarget. 2016; 7(52): 86011-86025, doi: 10.18632/oncotarget.13322, indexed in Pubmed: 27852068.

24. Qi CL, Wei Bo, Ye J, et al. P-selectin-mediated platelet adhesion promotes the metastasis of murine melanoma cells. PLoS One. 2014; 9(3): e91320, doi: 10.1371/journal.pone.0091320, indexed in Pubmed: 24632801.

25. Pulaski BA, Ostrand-Rosenberg S. Mouse 4T1 breast tumor model. Curr Protoc Immunol. 2001; Chapter 20: Unit 20.2, doi: 10.1002/0471142735.im2002s39, indexed in Pubmed: 18432775 .
26. Cuesta R, Holz MK. RSK-mediated down-regulation of PDCD4 is required for proliferation, survival, and migration in a model of triple-negative breast cancer. Oncotarget. 2016; 7(19): 27567-27583, doi: 10.18632/oncotarget.8375, indexed in Pubmed: 27028868.

27. Zhao H, Martin TA, Davies EL, Ruge F, Yu H, Zhang Y, et al. The Clinical Implications of RSK1-3 in Human Breast Cancer. Anticancer Res. 2016; 36(3): 1267-1274, indexed in Pubmed: 26977024.

28. Dehm SM, Bonham K. SRC gene expression in human cancer: the role of transcriptional activation. Biochem Cell Biol. 2004; 82(2): 263-274, doi: 10.1139/o03-077, indexed in Pubmed: 15060621.

29. Eisinger-Mathason TS, Andrade J, Groehler AL, et al. Codependent functions of RSK2 and the apoptosis-promoting factor TIA-1 in stress granule assembly and cell survival. Mol Cell. 2008; 31(5): 722-736, doi: 10.1016/j.molcel.2008.06.025, indexed in Pubmed: 18775331.

30. Eisinger-Mathason TS, Andrade J, Lannigan DA. RSK in tumorigenesis: connections to steroid signaling. Steroids. 2010; 75(3): 191-202, doi: 10.1016/j.steroids.2009.12.010, indexed in Pubmed: 20045011.

31. Chi Y, Huang S, Liu M, et al. Cyclin D3 predicts disease-free survival in breast cancer. Cancer Cell Int. 2015; 15: 89, doi: 10.1186/s12935-015-0245-6, indexed in Pubmed: 26412984.

32. Sicinska E, Aifantis I, Cam LLe, et al. Requirement for cyclin D3 in lymphocyte development and T cell leukemias. Cancer Cell. 2003; 4(6): 451-461, doi: 10.1016/s1535-6108(03)00301-5.

Submitted: 18 October, 2017

Accepted after reviews: 16 February, 2018 Available as AoP: 27 February, 2018 